\title{
Dynamics of on-board rotors on finite-length journal bearings subject to multi-axial and multi-frequency excitations: numerical and experimental investigations
} \author{
and Sophie Baudin ${ }^{2}$ \\ ${ }^{1}$ Univ Lyon, INSA Lyon, CNRS, LaMCoS, UMR5259, 69621 Villeurbanne, France \\ 2 AVNIR Engineering, 62 Boulevard Niels Bohr, 69603 Villeurbanne Cedex, France
}

Yvon Briend ${ }^{1, *}$, Eric Chatelet $^{1}$, Régis Dufour ${ }^{1}$, Marie-Ange Andrianoely ${ }^{1}$, Franck Legrand ${ }^{1}$,

Received: 15 January 2021 / Accepted: 30 April 2021

\begin{abstract}
On-board rotating machinery subject to multi-axial excitations is encountered in a wide variety of high-technology applications. Such excitations combined with mass unbalance forces play a considerable role in their integrity because they can cause parametric instability and rotor-stator interactions. Consequently, predicting the rotordynamics of such machines is crucial to avoid triggering undesirable phenomena or at least limiting their impacts. In this context, the present paper proposes an experimental validation of a numerical model of a rotor-shaft-hydrodynamic bearings system mounted on a moving base. The model is based on a finite element approach with Timoshenko beam elements having six degrees of freedom (DOF) per node to account for the bending, torsion and axial motions. Classical 2D rectangular finite elements are also employed to obtain the pressure field acting inside the hydrodynamic bearing. The finite element formulation is based on a variational inequality approach leading to the Reynolds boundary conditions. The experimental validation of the model is carried out with a rotor test rig, designed, built, instrumented and mounted on a 6-DOF hydraulic shaker. The rotor's dynamic behavior in bending, torsion and axial motions is assessed with base motions consisting of mono- and multi-axial translations and rotations with harmonic, random and chirp sine profiles. The comparison of the predicted and measured results achieved in terms of shaft orbits, full spectrums, transient history responses and power spectral densities is very satisfactory, permitting the experimental validation of the model proposed.
\end{abstract}

Keywords: On-board rotor / experimental validation / hydrodynamic bearings / 6-DOF shaker / multi-axial excitation / random motion

\section{Introduction}

Rotating machinery subject to base motions, so-called on-board rotor, is a common feature in many industrial fields. Examples include a helicopter turbo-engine subject to airflight maneuvers and spectral lines associated with rotor blades, a turbopropeller suspended from an aircraft wing excited by the aerodynamic forces of a broad frequency range, and a spatial turbopump undergoing pyrotechnic shocks. These systems fulfill vital functions that must be continuously ensured despite the internal and external excitations they undergo during their lifetime. Internal excitations include the unavoidable residual mass unbalances that generate forces synchronous with the rotation speed and which are responsible for resonance phenomena when passing through critical speeds. The external forces generated from the base motions are

\footnotetext{
* e-mail: yvon.briend@insa-lyon.fr
}

generally asynchronous and may create parametric excitation. In the presence of nonlinearities, these forces are able to trigger dynamic behaviors such as bifurcations, and quasi-periodic and chaotic motions. These phenomena may be sources of failures by fatigue $[1,2]$ or may lead to rotor-stator contacts, with the destruction of labyrinth seals or turbine blades [3]. In order to ensure the integrity of on-board rotors, predicting the emergence of such phenomena is crucial to ensure they can be avoided or that their impacts can at least be limited. This goal can be achieved by the development of reliable on-board rotor modeling, rigorously validated by a complete series of experimental comparisons.

In the last decade, contributions devoted to the topic of on-board rotors have been relatively abundant. In particular, Dakel et al. [4] analyzed the bending vibrations of a simply supported rotor composed of a shaft with one disk, excited by both its base and the shaft asymmetry. Their numerical results in terms of stability charts 
and shaft orbits evidenced the interactions between the two types of parametric excitations. Afterwards, the same authors [5] focused on the same shaft-disk system, this time symmetrical, mounted on short journal bearings with the great advantage of having an analytical solution to the Reynolds equation. The following year, the lateral and torsional dynamics of a multi-shaft on-board rotor was examined by Han and Chu [6]. Their time-history and spectral analysis revealed that the roll rotation of the base was responsible for a noticeable increase in system response and the occurrence of new resonances in the vicinity of the natural frequencies. Later, they determined in [7] the stability of a single-shaft rotor in bending only, parametrically excited by one or several harmonic rotations of its base. The influence of the phase between the combined base rotations acting with the same frequency was shown in stability charts. Thereafter, the non-stationary shaft's speed of rotation was introduced numerically in on-board rotor applications by Bouziani et al. [8], who performed a numerical analysis of the bending behavior in terms of spectrograms. Saimi et al. [9] used a new discretization method with "h-p" finite elements to model an on-board rotor with a cantilevered disk, undergoing constant base rotations which altered the Campbell diagram. Following the work done by Dakel et al. [4], onboard rotors supported by journal bearings have attracted much attention. Wang et al. [10] considered the case of a centrifugal pump modeled by a simplified six degrees of freedom (DOF) system and subject to the El Centro earthquake, Reddy et al. [11] introduced viscoelastic damping in the journal bearings of a rotor representing a turbocharger, and Liu et al. [12] compared two journal bearing models in terms of shaft orbits for a rotor with one disk excited by a harmonic base translation.

Several authors addressed support flexibility in combination with non-inertial support motion. Vicencio and Cruz [13] examined the dynamics of rotating machinery used in the nuclear field, such as gas and steam turbines supported by hydrodynamic bearings mounted on flexible foundations. By imposing base translations defined with accelerograms corresponding to shock response spectrums from real seismic excitations, they deduced the impact of the nonlinearities stemming from the journal bearings and the importance of knowing the foundation characteristics of such rotors. Zhu et al. [14] analyzed an on-board hollow rotor mounted on hydrodynamic bearings included within rubber damping rings showing viscoelastic behavior. They first demonstrated the apparent passive vibration control related to this additional damping, in particular in the vicinity of the critical speeds, and secondly the influence of the base translations on the whole rotor-bearing-foundation-system. Sales et al. [15] modeled the bending, torsional and longitudinal effects of a rotor mounted on a flexible moving support represented by a Timoshenko beam. The impact of support flexibility was exhibited on the bending effect through the disk orbits and the Campbell diagram for harmonic base rotations composed of roll and yaw.

Lastly, a novel control law intended to master the parametric instabilities of an on-board rotor was proposed by Soni et al., for a shaft mounted on active magnetic bearings [16] and in the presence of an electromagnetic actuator [17]. Other contributions were devoted to onboard rotors with geometric nonlinearities caused by large shaft displacements. This was the case of Phadatare et al. [18] with their highly flexible shaft mounted on a base subject to sinusoidal vertical translations and of Shahgholi et al. [19] with their asymmetric shaft whose support was subject to sinusoidal transverse rotations. In both these works, the shaft dynamics was restricted to the first mode of the linear system to facilitate the application of perturbation methods. As performed previously by Dakel et al. [4], Yi et al. [20] introduced the shaft and disk asymmetries of an on-board rotor and afterwards demonstrated the frequency combinations stemming from the rotational base motions and the asymmetry through the spectral displacement response of the disk. Qiu et al. [21] focused on the dynamics of a planetary gear excited by harmonic yaw motions of its support. Sousa et al. [22] and Stanica et al. [23] carried out several numerical investigations such as the analysis of the influence of a constant support rotation on the variation of critical speeds. Other simplified models have been developed recently to assess the impact of the wave motions on the engine rotors of maritime vessels [24,25].

All the research of this last decade mentioned previously has dealt exclusively with numerical results. The experimental investigations performed after those of Lee et al. [26], Duchemin et al. [27] and Driot et al. [28] are however much rarer. Among the few cases was the work done by Sousa et al. [29] who built an on-board rotor test bench composed of a slender shaft with a constant circular cross section and a disk supported by ball bearings. This rotor was excited by its base with a mono-axial electrodynamic shaker reproducing shock and sinusoidal base translations. Their numerical model was validated in terms of time-history disk displacements in the case of a shock profile for the rotor at rest and of a sinusoidal profile for the rotor operating at both sub- and supercritical speeds. Chen et al. [30] designed a support for combining simultaneous rotations around two orthogonal axes. Nonetheless, they excited their rotor with only one roll rotation of the base since the shaker they used was mono-axial. The validation of their model was carried out on a harmonic profile from the disk displacements in the time domain. More recently, Jarroux et al. [31] mounted a rotor equipped with active magnetic bearings on a 6DOF hydraulic shaker, the bearings being included within touch-down bearings with elastic dampers. This rotor was subjected to a harmonic vertical translation of its support for several acceleration levels until the occurrence of rotor-stator contacts within the active magnetic bearings. Their model was validated in terms of shaft orbits using numerical-experimental comparisons.

Therefore, strong interest in on-board investigations can be found in the literature. Nevertheless, most of the works remain numerical, with assumptions that are often far from those occurring in real industrial systems. This is the case for instance with the papers devoted to journal bearings $[5,10-13,24,25]$ which exploited the short bearing hypothesis so as to use analytical solutions of the Reynolds equation and thus facilitate the numerical investigations. 
Furthermore, the base excitations were still too restricted to mono-axial and mono-frequency cases as well as limited to the shaft bending dynamics without accounting for the axial and torsional effects [5,7-11,14]. This also holds for the experimental works, mostly owing to test benches using mono-axial shakers that moreover do not permit a straightforward change of position of the instantaneous axis of the base rotation. In addition, the experimental validations have been exclusively performed with shafts mounted on ball bearings rather than on hydrodynamic bearings for which numerical results are however abundant. The purpose of the present paper is thus to overcome all these issues by proposing a novel and complete experimental study in order to validate an on-board rotor model developed previously in [32]. The latter has six DOFs per node to account for all bending, torsion and axial shaft motions. The rotor is mounted on finite-length hydrodynamic bearings. Simultaneous translational and rotational base motions are properly defined to be able to correctly represent the multi-axial experimental excitations. Experimental validations of the linearized model are then carried out afterwards for one subcritical shaft speed of rotation, not only with mono-axial base excitations but also with multi-axial and multi-frequency base excitations. For the sake of completeness, several time profiles of base motions are imposed such as harmonic, random and chirp sine. The bending, axial and torsional shaft dynamics are all addressed successively. In order to obtain an experimental validation of the model proposed, a test rig of an on-board rotor has been designed, built and instrumented accordingly. A 6-DOF hydraulic shaker is used to subject the rotor to base excitations.

\section{Modeling and base motion definition}

A typical on-board rotor is sketched in Figure 1a. Two frames of reference are necessary to represent its dynamics. The first one, $\mathcal{R}_{0}\left(O_{0}, \vec{X}_{0}, \vec{Y}_{0}, \vec{Z}_{0}\right)$, is considered as Galilean. The second one, $\mathcal{R}(O, \vec{x}, \vec{y}, \vec{z})$, is fixed to the rotor base and thus independent of the shaft's rotation, with its origin $O$ located at the left end of the shaft's neutral axis and $\vec{y}$ along the shaft's axis of rotation at rest. A complete description and model of the rotor dynamics by a finite element method was proposed in [32]. By way of a brief recall, the shaft is modeled with 1D Timoshenko beam elements having two nodes and six DOFs per node which are $(u, w)$ the bending transverse displacements along $(\vec{x}, \vec{z})$ respectively, $v$ the axial displacement in $\vec{y},(\psi, \theta)$ the bending transverse rotations around $(\vec{z}, \vec{x})$ respectively, and $\beta$ the torsion angle of rotation around $\vec{y}$. All these displacements depend on only the axial coordinate $y$, and the total shaft speed of rotation $\dot{\phi}$ is defined as $\dot{\phi}(y)=\dot{\phi}^{*}+\dot{\beta}(y)$ where $\dot{\phi}^{*}$ is the constant nominal shaft speed of rotation provided by the motor. Cubic shape functions are employed to approximate $(u, w)$ while linear shape functions are used for $v$ and $\beta$. The following system of equations governing the rotor dynamics can be reached in a matrix form by applying the Lagrange's equations to all the energies and virtual works of the system [32]:

$$
\begin{aligned}
& \mathbf{M} \ddot{\boldsymbol{\delta}}+\left(\mathbf{G}+\mathbf{C}_{\mathbf{j b}}+\mathbf{C}_{\mathbf{b a}}\right) \dot{\boldsymbol{\delta}}+\left(\mathbf{K}+\mathbf{K}_{\mathbf{j b}}+\mathbf{K}_{\mathbf{b a}}\right) \boldsymbol{\delta} \\
& =\mathbf{F}_{\mathbf{g l}}+\mathbf{F}_{\mathbf{u}}+\mathbf{F}_{\mathbf{b a}}
\end{aligned}
$$

with $\mathbf{M}, \mathbf{G}$ and $\mathbf{K}$ being the classical mass, gyroscopic and structural stiffness matrices, respectively, $\mathbf{F}_{\mathbf{u}}$ the classical mass unbalance force vector and $\boldsymbol{\delta}$ the global DOF vector expressed with respect to the static equilibrium position. $\mathbf{C}_{\mathbf{b a}}, \mathbf{K}_{\mathbf{b a}}$ and $\mathbf{F}_{\mathbf{b a}}$ are the time-dependent damping and stiffness matrices and the force vector, respectively, related to the base motion. $\mathbf{F}_{\mathbf{g l}}$ is a force vector related to the gravity load oriented along $\vec{X}_{0}$, which is non-zero and time-dependent only in the case of a rotational base motion [32] (pitch or roll only, given here the gravity is along the yaw axis). It is obtained as $\mathbf{F}_{\mathbf{g l}}=\mathbf{F}_{\mathbf{g d}}-\mathbf{F}_{\mathbf{g s}}$ where $\mathbf{F}_{\mathbf{g d}}$ is the full dynamic gravity load vector and $\mathbf{F}_{\mathbf{g s}}$ is the static (without base motion) gravity load vector. The remaining terms are related to the linearization of the journal bearing restoring forces in the vicinity of the static equilibrium position at a given constant shaft speed of rotation characterized by the static solution vector $\boldsymbol{\delta}_{\boldsymbol{s}}$. In this context, $\mathbf{C}_{\mathbf{j b}}$ and $\mathbf{K}_{\mathbf{j b}}$ are respectively the damping and stiffness matrices derived from these restoring forces with respect to $\dot{\boldsymbol{\delta}}$ and $\boldsymbol{\delta}$. The computation of the pressure fields providing the restoring forces of the journal bearings are obtained using a 2D FEM with 4-node rectangular elements, taking into account the cavity problem by applying the Reynolds boundary conditions as in [33]. The only damping is provided by either the hydrodynamic bearings or the motor-shaft coupling. Unlike in [32], non-stationary effects are not accounted for. Furthermore, the mass unbalance is considered small enough so that the additional matrices related to mass unbalance are neglected.

Given the aim of the present paper to deal with multiaxial excitations, a complete description of the rotor base kinematics is essential, especially in the case of combined translational and rotational motions where the rotations are defined around a fixed axis that may not necessarily pass through $O$. Let the base be rigid but mobile. To begin with the rotations only, the orientation of the moving frame $\mathcal{R}$ with respect to $\mathcal{R}_{0}$ is done with a classical approach for rigid solids using the Euler angles. Thus, $\mathcal{R}$ is obtained from $\mathcal{R}_{0}$ by three successive rotations: a pitch rotation of angle $\alpha_{1}$ around $Z_{0}$ that creates a first intermediary frame $\mathcal{R}_{1}\left(O_{0}, \vec{X}_{1}, \vec{Y}_{1}, \vec{Z}_{1}\right)$ with $\vec{Z}_{1}=\vec{Z}_{0}$, a yaw rotation of angle $\alpha_{2}$ around $\vec{X}_{1}$ that creates a second intermediary frame $\mathcal{R}_{2}\left(O_{0}, \vec{X}_{2}, \vec{Y}_{2}, \vec{Z}_{2}\right)$ with $\vec{X}_{2}=\vec{X}_{1}$, and a third roll rotation of angle $\alpha_{3}$ around $\vec{Y}_{2}$ that creates $\mathcal{R}$ with $\vec{Y}_{2}=\vec{y}$. The translation motion of $\mathcal{R}$ with respect to $\mathcal{R}_{0}$ is defined through the motion of the origin $O$ of $\mathcal{R}$ in $\mathcal{R}_{0}$ by the vector $\overrightarrow{O_{0} O}=X_{0} \vec{X}_{0}+Y_{0} \vec{Y}_{0}+Z_{0} \vec{Z}_{0}$.

In order to now impose base motions composed of simultaneous translations and rotations, one possible approach decomposes the variables $\left(X_{0}, Y_{0}, Z_{0}\right)$ such that:

$$
X_{0}=X_{0, r o t}+X_{0, t r}
$$




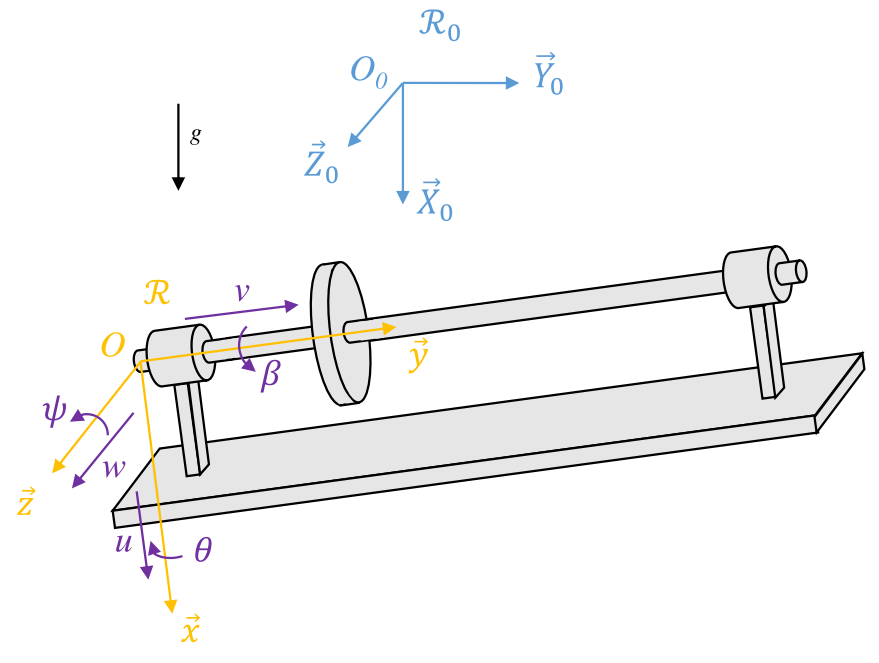

(a)

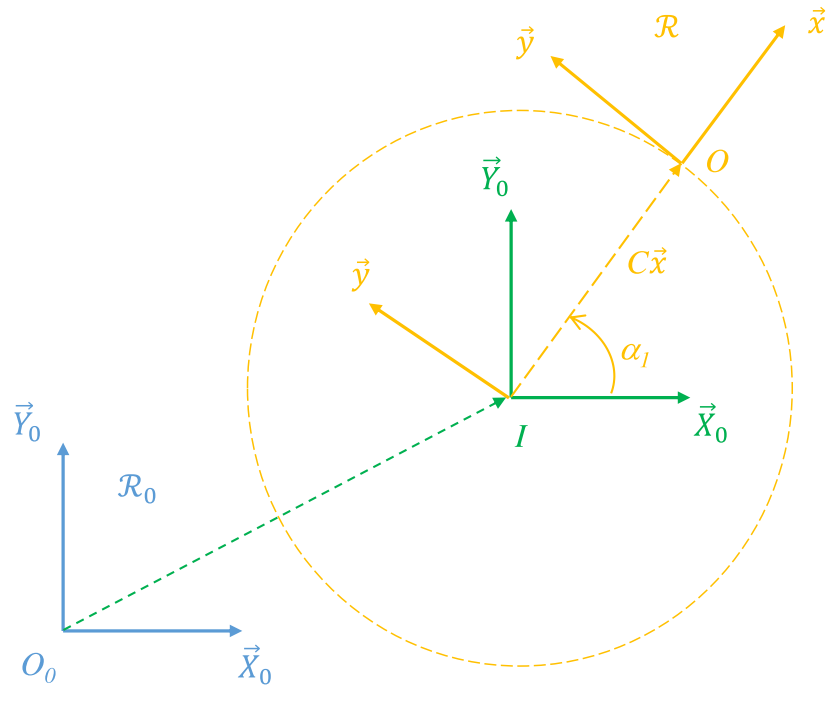

(b)

Fig. 1. (a) Sketch of an on-board rotor and (b) Example of an in-plane motion of the moving frame $\mathcal{R}$, composed of two translations along $\vec{X}_{0}$ and $\vec{Y}_{0}$ and a rotation of angle $\alpha_{1}$ and radius $C$.

$$
\begin{aligned}
& Y_{0}=Y_{0, r o t}+Y_{0, t r} \\
& Z_{0}=Z_{0, r o t}+Z_{0, t r}
\end{aligned}
$$

where $\left(X_{0, \text { rot }}, Y_{0, \text { rot }}, Z_{0, \text { rot }}\right)$ and $\left(X_{0, t r}, Y_{0, t r}, Z_{0, t r}\right)$ are the variations of $\left(X_{0}, Y_{0}, Z_{0}\right)$ related to pure rotational and translational motions, respectively. In this way, it is possible to interpret the whole motion as a combination of a translation in $\mathcal{R}_{0}$ of a fictitious point $I$ and a rotation around a combination of the axes $\left(\vec{Z}_{0}, \vec{X}_{1}, \vec{Y}_{2}\right)$ passing through this point $I$. This is illustrated in Figure $1 \mathrm{~b}$ with an in-plane example composed of two translations along $\vec{X}_{0}$ and $\vec{Y}_{0}$ and a rotation around $\vec{Z}_{0}$. The previous variables $\left(X_{0, \text { rot }}, Y_{0, \text { rot }}, Z_{0, \text { rot }}\right)$ are then related to the constant coordinates $\left(x_{I}, y_{I}, z_{I}\right)$ of $I$ in the moving frame $\mathcal{R}$ by:

$$
\left\{\begin{array}{l}
X_{0, r o t} \\
Y_{0, \text { rot }} \\
Z_{0, \text { rot }}
\end{array}\right\}_{\mathcal{R}_{0}}=-\mathbf{P}_{\mathcal{R}_{\mathbf{0}} \rightarrow \mathcal{R}}\left\{\begin{array}{l}
x_{I} \\
y_{I} \\
z_{I}
\end{array}\right\}_{\mathcal{R}}
$$

while the variables $\left(X_{0, t r}, Y_{0, t r}, Z_{0, t r}\right)$ represent the coordinates of $I$ in $\mathcal{R}_{0}$ with $\mathbf{P}_{\mathcal{R}_{0} \rightarrow \mathcal{R}}$ the orthogonal transformation matrix from $\mathcal{R}_{0}$ to $\mathcal{R}$ in which appear cosine and sine functions of the angles $\left(\alpha_{1}, \alpha_{2}, \alpha_{3}\right)$. If $\left(X_{0, t r}, Y_{0, t r}, Z_{0, t r}\right)$ are nil or constant, then the actual instantaneous axis of rotation of $\mathcal{R}$ with respect to $\mathcal{R}_{0}$ passes through $I$.

Therefore, according to the previous approach, the complete motion of $\mathcal{R}$ is defined by three sets of parameters: (1) $\left(X_{0, t r}(t), Y_{0, t r}(t), Z_{0, t r}(t)\right)$ the variables that produce the translational motion along the fixed axes of the Galilean frame $\mathcal{R}_{0}$, referred to as pure translations in the sequel; (2) $\left(\alpha_{1}, \alpha_{2}, \alpha_{3}\right)$ the Euler angles that produce the rotational motion of $\mathcal{R}$ and all fixed points in $\mathcal{R}$ around an axis passing through point $I ;(3)\left(x_{I}, y_{I}, z_{I}\right)$ the constant coordinates of $I$ in $\mathcal{R}$. The matrices $\mathbf{C}_{\mathbf{b a}}, \mathbf{K}_{\mathbf{b a}}$ and vector $\mathbf{F}_{\mathbf{b a}}$ of equation (1) will then depend on these variables, as can be found in [32].

\section{Experimental validation}

\subsection{Presentation of the on-board rotor test bench}

As mentioned in Section 1, experimental investigations of on-board rotors are not abundant in the literature, even less so when the shaft is supported by hydrodynamic bearings and subject to multi-axial motions. Nonetheless, this type of bearing is widely used in industry and the excitations experienced by on-board rotating machines are often naturally multi-axial. These reasons thus motivated the design and fabrication of the rotor test bench presented in Figure 2. This rotor is composed of a steel shaft, two steel disks, two hydrodynamic bearings, a flexible coupling and a DC electric motor providing a maximum torque of 0.177 N.m. All the numerical data are available in Table 1. The whole system is mounted on a thick plate, itself fixed to the upper face of the 6-DOF hydraulic shaker driven in closed loop by means of accelerations. The latter is a cube-shaped long-stroke shaker, with an edge of $0.8128 \mathrm{~m}$ and offering accelerations up to $10 \mathrm{~g}$ within a frequency range of $[0-250] \mathrm{Hz}$. The motor-shaft coupling (model 321.12.2222 of Huco ${ }^{\circledR}$ ) characteristics are available in Table 2. These characteristics come either from the manufacturer or from an optimization performed through preliminary modal analysis with a fixed base.

Before any motion occurs, the Galilean frame $\mathcal{R}_{0}$ always has the same orientation as the moving frame $\mathcal{R}$ so that the angles $\left(\alpha_{1}, \alpha_{2}, \alpha_{3}\right)$ do not present any non-zero $0-\mathrm{Hz}$ static components. However, the location of $I$ in $\mathcal{R}$ may depend on the test. In the sequel, unless otherwise specified, it is set by $x_{I}=y_{I}=z_{I}=0$. The shaft is discretized 


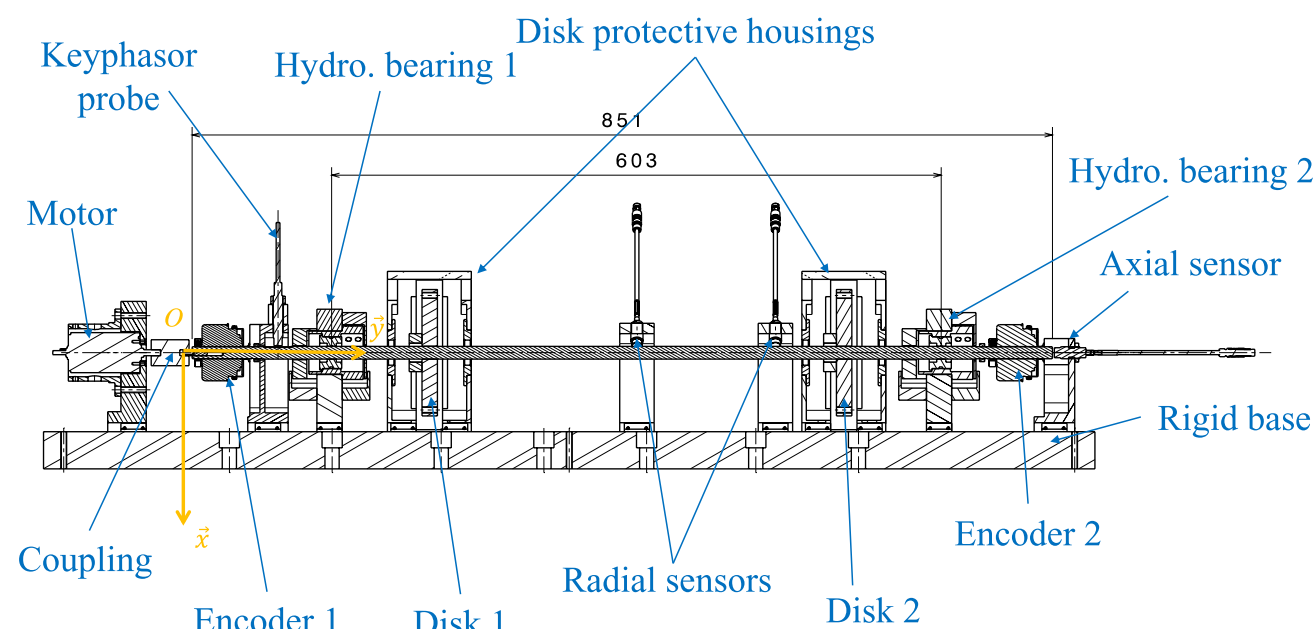

(a)

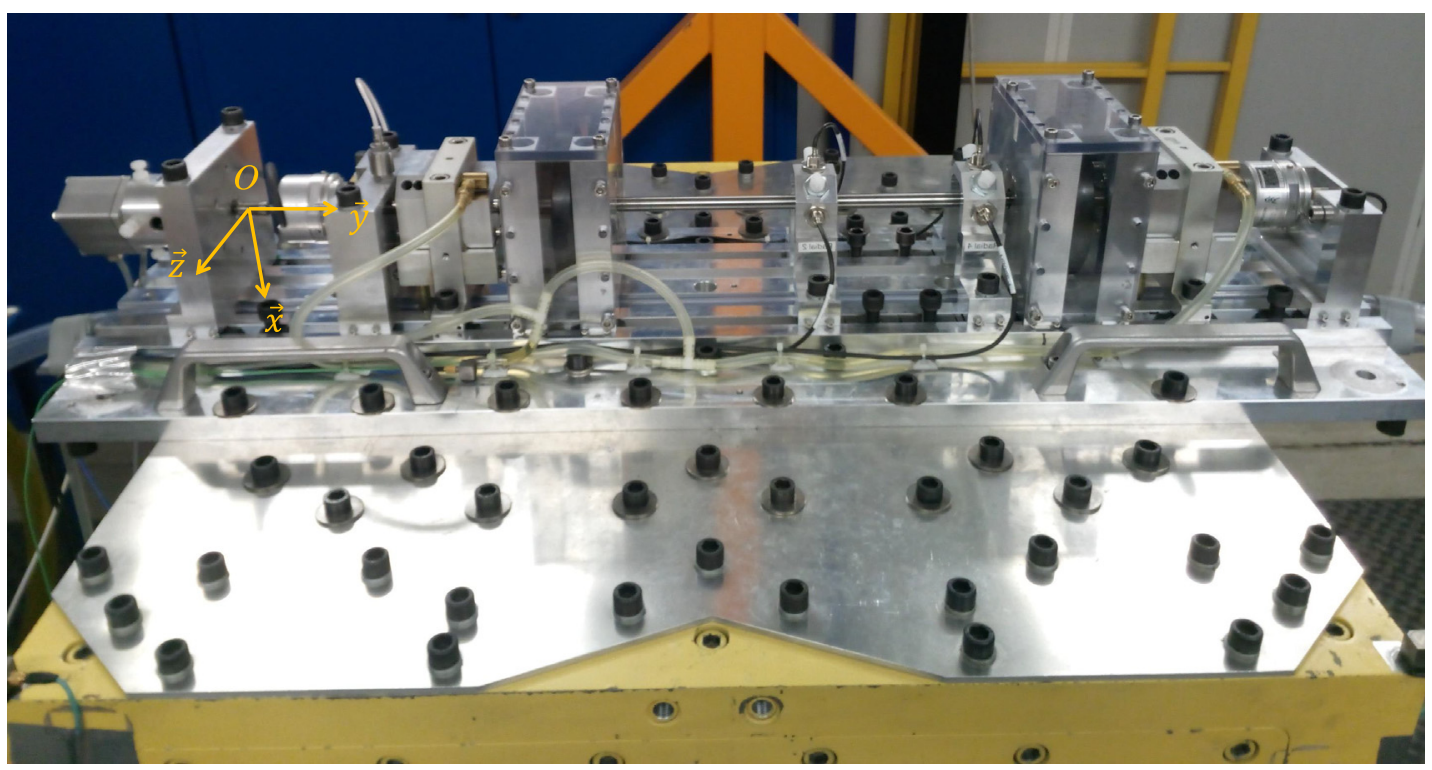

(b)

Fig. 2. On-board rotor test bench used for the experimental validations: (a) CAD view and (b) Photo view.

Table 1. Main rotor data.

\begin{tabular}{ll}
\hline Shaft length and main diameter & $0.851 \mathrm{~m} ; 12.7 \mathrm{~mm}$ \\
Shaft Young modulus and Poisson coefficient & $210 \mathrm{GPa} ; 0.3$ \\
Shaft and disk density & $7778 \mathrm{~kg} / \mathrm{m}^{3}$ \\
Radius and thickness of disks & $6.3055 \times 10^{-2} \mathrm{~m} ; 1.56 \times 10^{-2} \mathrm{~m}$ \\
Hydrodynamic bearing radius, length and clearance & $6.39 \times 10^{-3} \mathrm{~m} ; 6.2 \times 10^{-3} \mathrm{~m} ; 4 \times 10^{-5} \mathrm{~m}$ \\
Hydrodynamic bearing oil viscosity, feeding pressure and feeding hole radius & $0.0206 \mathrm{Pas} ; 4 \times 10^{4} \mathrm{~Pa} ; 1.25 \mathrm{~mm}$ \\
Mass unbalance value, position, radius and initial phase & $2.37 \mathrm{~g} ;$ Node $21 ; 57 \mathrm{~mm} ; 0^{\circ}$ \\
\hline
\end{tabular}

into 27 beam finite elements, as shown in Figure 3. The shaft radius is $6.35 \mathrm{~mm}$ for the FEs 4 to 24 and $6 \mathrm{~mm}$ for the FEs $(1,2,3,25,26,27)$. In order to model the shaft local stiffening due to the tightening of the disks on the shaft, angular stiffness coefficients of $100 \mathrm{N.m} / \mathrm{rad}$ are added to the coefficients related to the DOFs $\psi$ and $\theta$ of Nodes 9 and 21 in the structural stiffness matrix $\mathbf{K}$ of equation (1). These new coefficients were found by a preliminary modal analysis, the shaft having freefree boundary conditions, and proved to provide better agreements with the experimental results even with the supported shaft. The hydrodynamic bearings have finite 


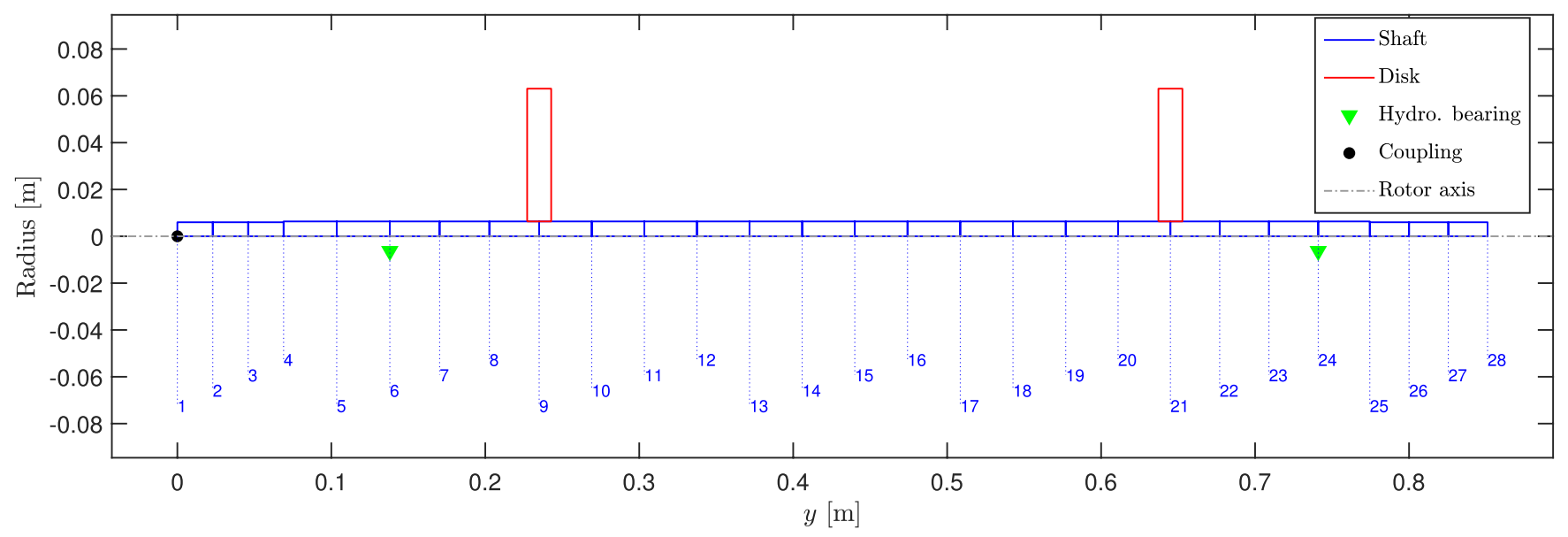

Fig. 3. On-board rotor FE mesh.

Table 2. Motor-shaft coupling characteristics of the rotor test bench.

\begin{tabular}{llll}
\hline Stiffness & Value & Damping & Value \\
\hline$K_{u u}$ & $1000 \mathrm{~N} / \mathrm{m}$ & $C_{u u}$ & $12 \mathrm{~N} \mathrm{~s} / \mathrm{m}$ \\
$K_{v v}$ & $2700 \mathrm{~N} / \mathrm{m}$ & $C_{v v}$ & $10 \mathrm{~N} \mathrm{~s} / \mathrm{m}$ \\
$K_{w w}$ & $1000 \mathrm{~N} / \mathrm{m}$ & $C_{w w}$ & $12 \mathrm{~N} \mathrm{~s} / \mathrm{m}$ \\
$K_{\theta \theta}$ & $0.21 \mathrm{~N} . \mathrm{m} / \mathrm{rad}$ & $C_{\theta \theta}$ & $1 \times 10^{-3} \mathrm{Nms} / \mathrm{rad}$ \\
$K_{\psi \psi}$ & $0.21 \mathrm{Nm} / \mathrm{rad}$ & $C_{\psi \psi}$ & $1 \times 10^{-3} \mathrm{N.m.s} / \mathrm{rad}$ \\
$K_{\beta \beta}$ & $27 \mathrm{Nm} / \mathrm{rad}$ & $C_{\beta \beta}$ & $1 \times 10^{-2} \mathrm{Nms} / \mathrm{rad}$ \\
\hline
\end{tabular}

lengths with a length/diameter ratio of 0.4851 . They are fed by two circular feeding holes located in the axial middle at $0^{\circ}$ and $180^{\circ}$ with respect to $\vec{z}$. The hydrodynamic bearings are discretized using a $2 \mathrm{D}$ rectangular mesh with 200 nodes and 15 nodes in the circumferential and axial directions, respectively. The linearized stiffness and damping matrices at the operating speed $\dot{\phi}^{*}=1700 \mathrm{rpm}$ are summed in Table 3.

In order to assess the whole shaft dynamics, the rotor is equipped with several types of sensor. Firstly, four radial proximity probes (inductive sensors with eddy current) are distributed in two probe supports to monitor the shaft bending motion. These supports are localized at Nodes 15 and 19, i.e. relatively far from the bearings, because of constraints regarding the available space and to maximize the measurement amplitudes. Each probe was rigorously calibrated with respect to the specific circular shape and material of the shaft. These sensors are directed along either $\vec{x}$ or $\vec{z}$ of $\mathcal{R}$. Secondly, an axial sensor of the same model is set on one shaft end (Node 28 ) in order to measure the axial rigid-body motion. Thirdly, two optical encoders with a resolution of 4096 line counts are installed at Nodes 2 and 27 to access the shaft torsional motion. The stator part of these sensors is not fixed to the stator nor any support so as to avoid additional localized stiffness of the shaft. Their weight of $215 \mathrm{~g}$ each is then fully supported by the shaft itself and they can follow its deflection so that their presence affects the original bending dynamics. However, the rotation of the stator part is prevented with an axial screw.
These encoders are only used and mounted on the shaft for the test in Section 3.4. Finally, another proximity probe directed along $\vec{x}$ is used as a keyphasor probe (tachometer) which detects an axial groove located on the shaft near Node 4. Regarding the 6-DOF shaker, it is equipped with six mono-axial accelerometers for its driving process and with four tri-axial accelerometers to retrieve the real shaker motion to be introduced into the on-board rotor MEF for validation purposes. The process to obtain this real 6-DOF motion is described in-depth in [34].

The Campbell diagram of the rotor test bench on a fixed base and without encoders is computed and presented in Figure 4. The tracking of the mode shapes is done by applying the $\mathrm{NC}^{2} \mathrm{O}$ criterion [35]. The solid, dashed and dash-dotted curves refer to the bending, axial and torsional modes, respectively. The first four critical speeds are $[2084,2281,4749,5402] \mathrm{rpm}$. The natural frequencies and the modal damping factors of each mode appearing in the Campbell diagram at $\dot{\phi}^{*}=1700 \mathrm{rpm}$ are listed in Table 4. The first two modes of the latter, i.e. the axial and torsion ones, are almost only rigid-body modes with a main localized deformation in the coupling. The higher modes, after the seventh one, are not displayed since their natural frequencies are higher than $250 \mathrm{~Hz}$ which is above the frequency range under study for the following test. As could be expected, the additional mass from the encoders tends to lower the natural frequencies related to bending and axial dynamics.

Regarding the further experimental validations, the shaft is rotating in the direct sense, i.e. from $\vec{z}$ to $\vec{x}$, at a constant nominal speed of $\dot{\phi}^{*}=1700 \mathrm{rpm}$. The keyphasor and proximity probe signals have the same sampling frequency, fixed to $4096 \mathrm{~Hz}$, in order to accurately determine the shaft angle of rotation at any time. Thus, the shaft angular position as well as the phase between mass unbalance and base motion can be introduced in the numerical model. The initial phases of the mass unbalance and the base motions are given with respect to the keyphasor pulse. Finally, except for the torsion measurements in Section 3.4, the measurements presented below are not filtered or processed with any data processing tools (including windowing). 
Table 3. Non-zero coefficients of the linearized stiffness and damping matrices of the hydrodynamic bearings at $\dot{\phi}^{*}=1700 \mathrm{rpm}$.

\begin{tabular}{lrrlrr}
\hline Stiffness & \multicolumn{1}{l}{ HB1 } & HB2 & Damping & \multicolumn{1}{l}{ HB1 } & HB2 \\
\hline$K_{u u}$ & $4.41 \times 10^{6} \mathrm{~N} / \mathrm{m}$ & $4.11 \times 10^{6} \mathrm{~N} / \mathrm{m}$ & $C_{u u}$ & $2.33 \times 10^{4} \mathrm{~N} \mathrm{~s} / \mathrm{m}$ & $2.19 \times 10^{4} \mathrm{~N} \mathrm{~s} / \mathrm{m}$ \\
$K_{u w}$ & $-2.45 \times 10^{6} \mathrm{~N} / \mathrm{m}$ & $-2.33 \times 10^{6} \mathrm{~N} / \mathrm{m}$ & $C_{u w}$ & $-5.66 \times 10^{3} \mathrm{~N} \mathrm{~s} / \mathrm{m}$ & $-5.40 \times 10^{3} \mathrm{~N} \mathrm{~s} / \mathrm{m}$ \\
$K_{w u}$ & $-4.39 \times 10^{5} \mathrm{~N} / \mathrm{m}$ & $-4.11 \times 10^{5} \mathrm{~N} / \mathrm{m}$ & $C_{w u}$ & $-5.66 \times 10^{3} \mathrm{~N} \mathrm{~s} / \mathrm{m}$ & $-5.40 \times 10^{3} \mathrm{~N} \mathrm{~s} / \mathrm{m}$ \\
$K_{w w}$ & $8.96 \times 10^{5} \mathrm{~N} / \mathrm{m}$ & $8.62 \times 10^{5} \mathrm{~N} / \mathrm{m}$ & $C_{w w}$ & $2.95 \times 10^{3} \mathrm{~N} \mathrm{~s} / \mathrm{m}$ & $2.86 \times 10^{3} \mathrm{~N} \mathrm{~s} / \mathrm{m}$ \\
& & & $C_{u \beta}$ & $0.1079 \mathrm{~N} \mathrm{~s} / \mathrm{rad}$ & $0.1032 \mathrm{~N} \mathrm{~s} / \mathrm{rad}$ \\
& & & $C_{w \beta}$ & $-0.0029 \mathrm{~N} \mathrm{~s} / \mathrm{rad}$ & $-0.0030 \mathrm{~N} \mathrm{~s} / \mathrm{rad}$ \\
\hline
\end{tabular}

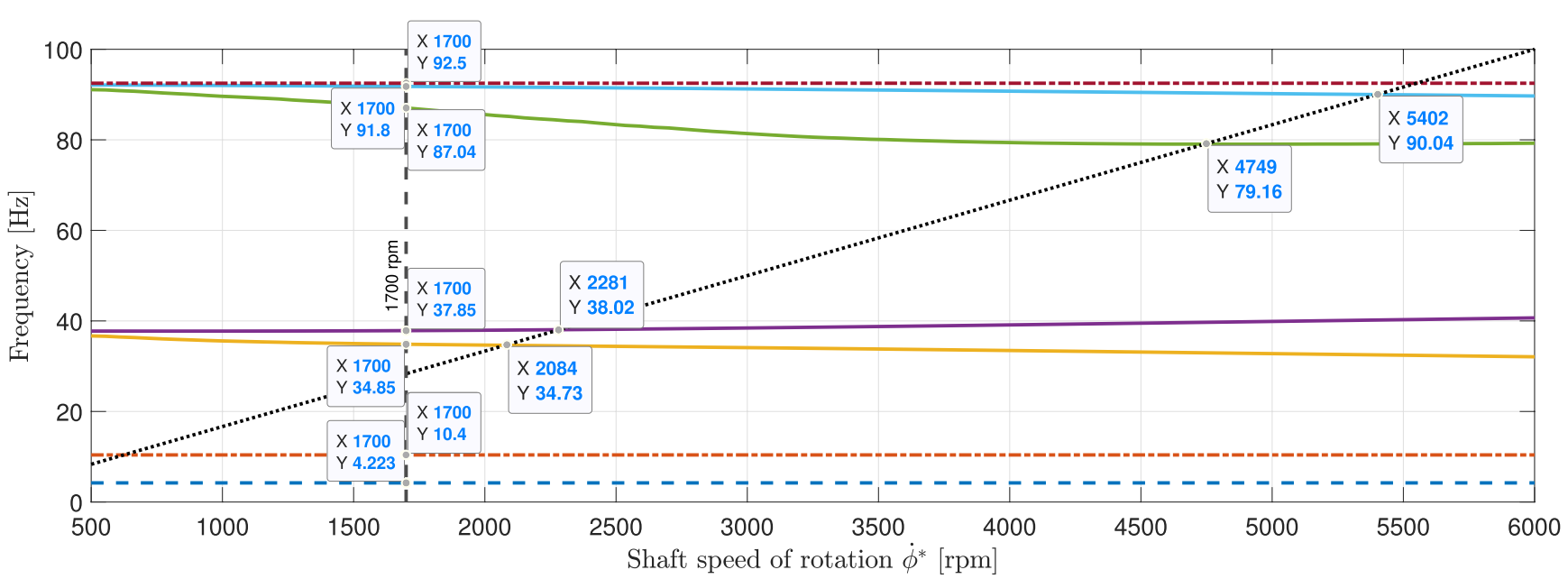

Fig. 4. Campbell diagram of the rotor test bench.

Table 4. Seven first numerical modes of the rotor test bench at $\dot{\phi}^{*}=1700 \mathrm{rpm}$ (FW: forward whirl, BW: backward whirl, WE: with encoders, ØE: without encoders).

\begin{tabular}{lcccll}
\hline Mode number & $\begin{array}{l}\text { Frequency } \\
{[\mathbf{H z}](\emptyset \mathbf{E})}\end{array}$ & $\begin{array}{l}\text { Modal damping } \\
\text { factor [\%] }(\emptyset \mathbf{E})\end{array}$ & $\begin{array}{l}\text { Frequency } \\
(\mathbf{W E})\end{array}$ & $\begin{array}{l}\text { Modal damping } \\
\text { factor [\%] }(\mathbf{W E})\end{array}$ & Modal shape \\
\hline 1 & 4.22 & 4.93 & 4.00 & 4.67 & Axial \\
2 & 10.40 & 1.16 & 10.40 & 1.16 & Torsion \\
3 & 34.85 & 3.87 & 33.63 & 2.89 & Bending (BW) \\
4 & 37.85 & 1.84 & 36.05 & 1.82 & Bending (FW) \\
5 & 87.04 & 11.39 & 82.18 & 4.02 & Bending (FW) \\
6 & 91.80 & 1.57 & 82.62 & 1.43 & Bending (BW) \\
7 & 92.50 & 0.14 & 92.50 & 0.14 & Torsion \\
\hline
\end{tabular}

\subsection{Multi-axial harmonic excitation}

The first case of base excitation focuses on multi-axial harmonic translations and on bending dynamics. The motion comprises two translations: the first one along $\vec{X}_{0}$ defined such that $X_{0, t r}=A_{X} \cos \left(2 \pi f_{X} t+\varphi_{X}\right)$ with $A_{X}=$ $15 \mu \mathrm{m}, f_{X}=42.5 \mathrm{~Hz}$ and $\varphi_{X}=57^{\circ}$ and the second one along $\vec{Z}_{0}$ defined such the $Z_{0, t r}=A_{Z} \cos \left(2 \pi f_{Z} t+\varphi_{Z}\right)$ with $A_{Z}=15 \mu \mathrm{m}, f_{Z}=56.7 \mathrm{~Hz}$ and $\varphi_{Z}=91^{\circ}$. The frequencies were chosen intentionally as harmonics of the shaft speed of rotation frequency $\mathrm{X}\left(f_{X} \equiv 1.5 \mathrm{X}\right.$ and $\left.f_{Z} \equiv 2 \mathrm{X}\right)$ to obtain a periodic shaft dynamics and periodic orbits. In fact, the shaft speed of rotation delivered by the motor is slightly lower than $\dot{\phi}^{*}=1700 \mathrm{rpm}$, inducing a slow variation of the experimental orbits with time. This may be interpreted as a variation of the phase between the mass unbalance and the base motion.
The raw (without filter) experimental and numerical orbits of Nodes 15 and 19 are shown in Figure 5a and Figure 5b, respectively, within a time interval of $2 \mathrm{~s}(\approx 57$ full shaft rotations). In addition, the shaft position at the experimental and numerical keyphasor pulses is superimposed on the respective shaft orbits. Excellent agreement can be noticed between the two sets of results in terms of orbit shape, amplitude and phase. Furthermore, the shaft occupies the same position at the keyphasor pulses (two pulses are seen in the orbits since a period is obtained for two full shaft rotations owing to the $1 \mathrm{X}$ frequency of excitation). However, some small deviations may be noted in amplitude in both Nodes 15 and 19. This can be attributed mainly to the presence of a residual mass unbalance since initial shaft balancing is never perfect, but also to some likely weaknesses in the modeling of the hydrodynamic bearings. It is noteworthy that the two orbits of Nodes 15 and 19 are relatively similar to one another. This is 


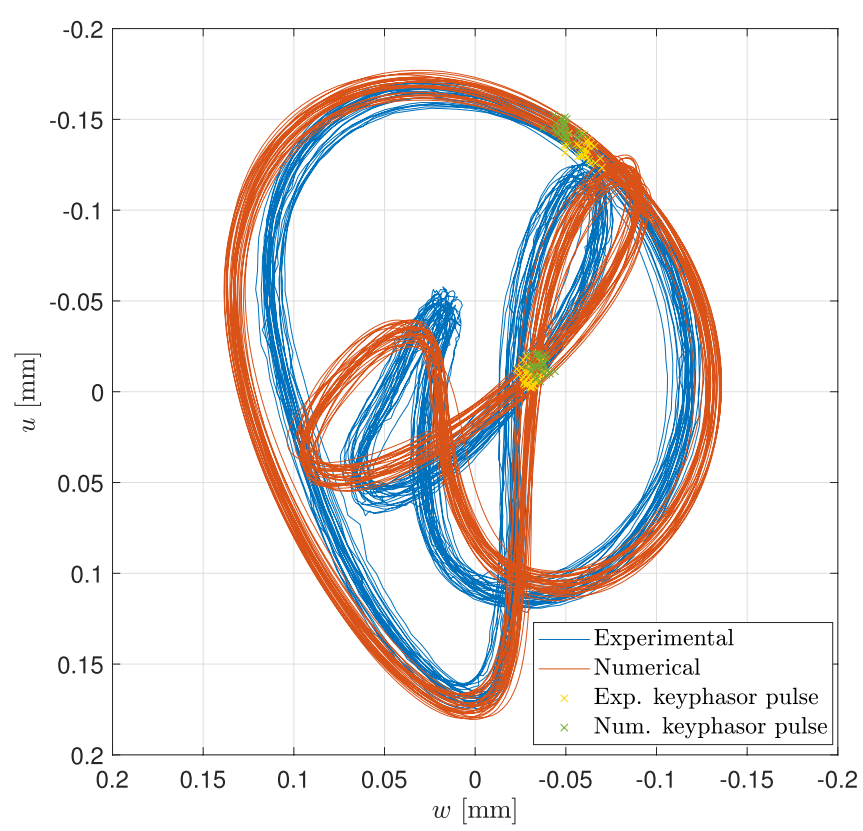

(a)

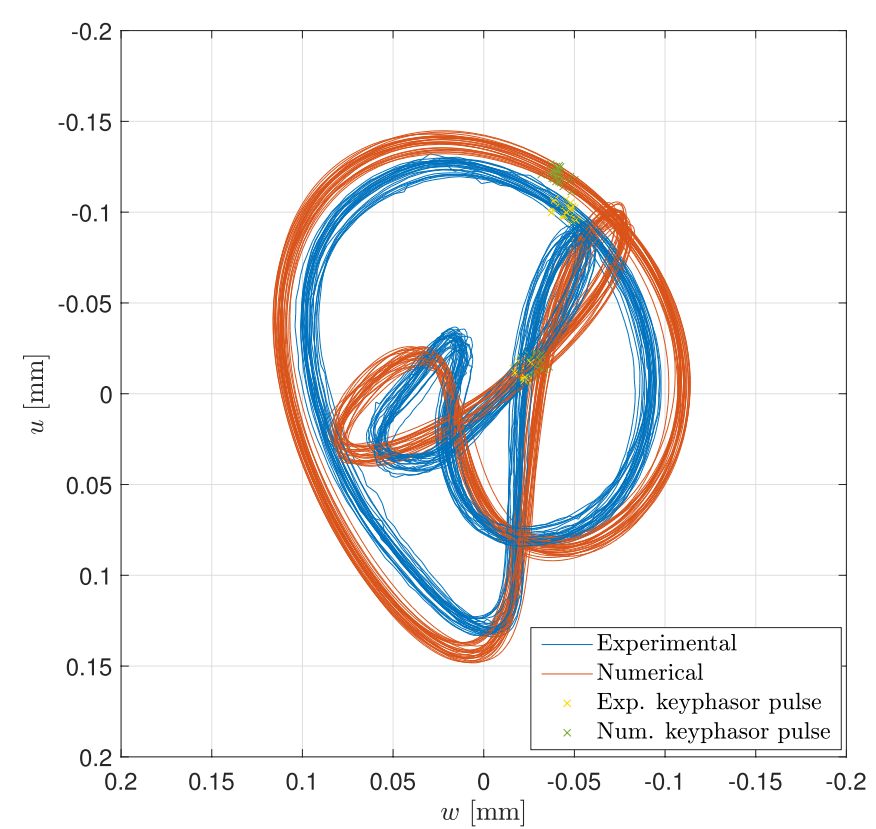

(b)

Fig. 5. Experimental-numerical comparison of shaft orbits in response to mass unbalance and two harmonic translations along $\vec{X}_{0}$ and $\vec{Z}_{0}$ within a time interval of $2 \mathrm{~s}$ ( $\approx 57$ full shaft rotations): (a) Node 15 and (b) Node 19.

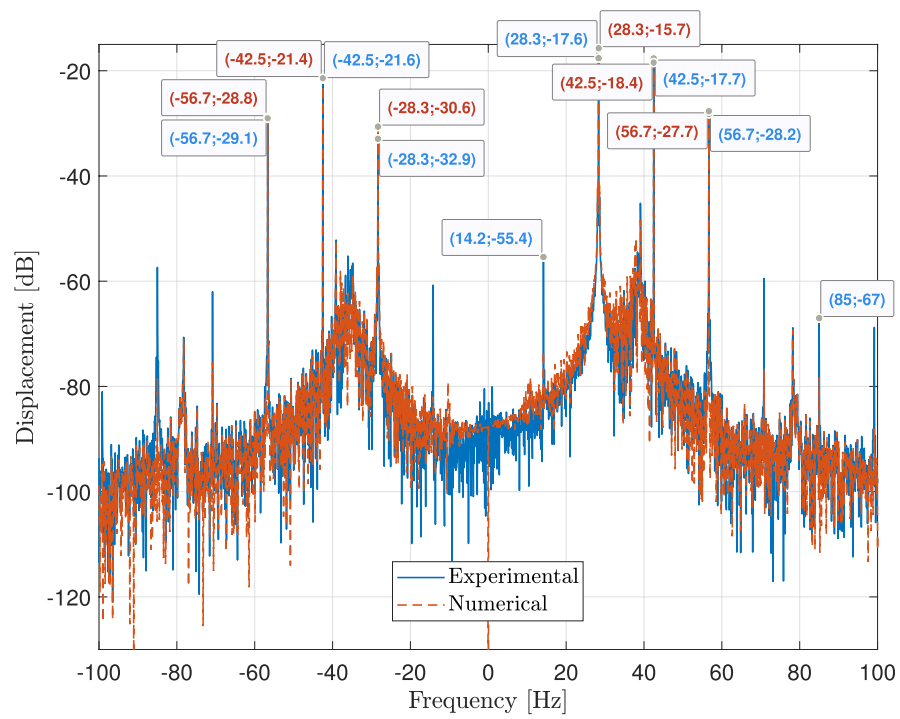

(a)

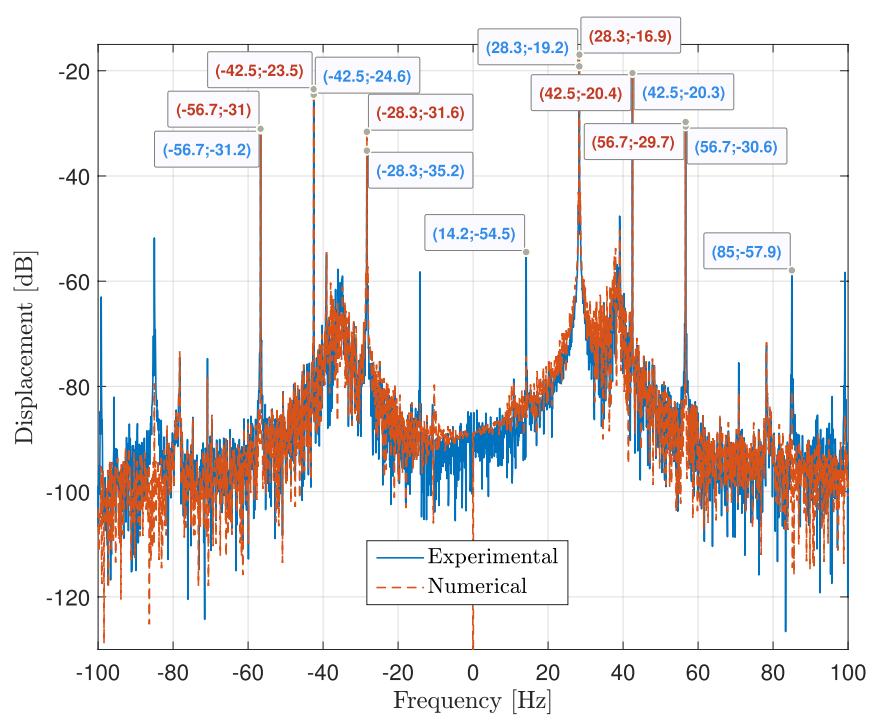

(b)

Fig. 6. Full spectrum of the numerical and experimental orbits of Figure 5: (a) Node 15 and (b) Node 19.

due to the fact that the frequencies of the base translations involved tend to excite the first two bending modes more (Modes 3 and 4 of Tab. 4 which are characterized by a maximum displacement in the middle of the shaft). Given the position of the radial probes supports, these modes do not induce any phase shift between these two orbits.

The full spectrum [36] of these experimental and numerical orbits are shown in Figure 6. They are obtained from the time signal on $12 \mathrm{~s}$ once the steady state is reached and normalized by the signals length $(=12 \mathrm{~s} \times 4096 \mathrm{~Hz})$. On looking at these new results, it can be seen that the accuracy of the numerical model is also established in the frequency domain. Besides, the amplitude discrepancy of the orbits of Nodes 15 and 19 mostly occurs at the shaft speed rotation frequency $( \pm 28.3 \mathrm{~Hz})$ rather than on the base motion frequencies. This might mean that this discrepancy is due more to the residual mass unbalance than to weaknesses in the modeling of the hydrodynamic bearings. Other deviations may be noticed in some harmonics 


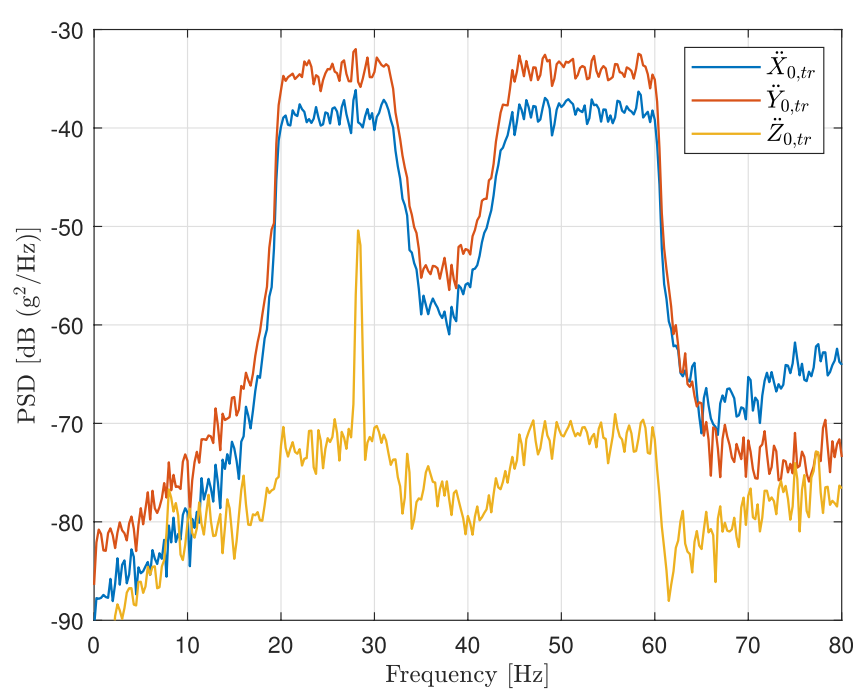

(a)

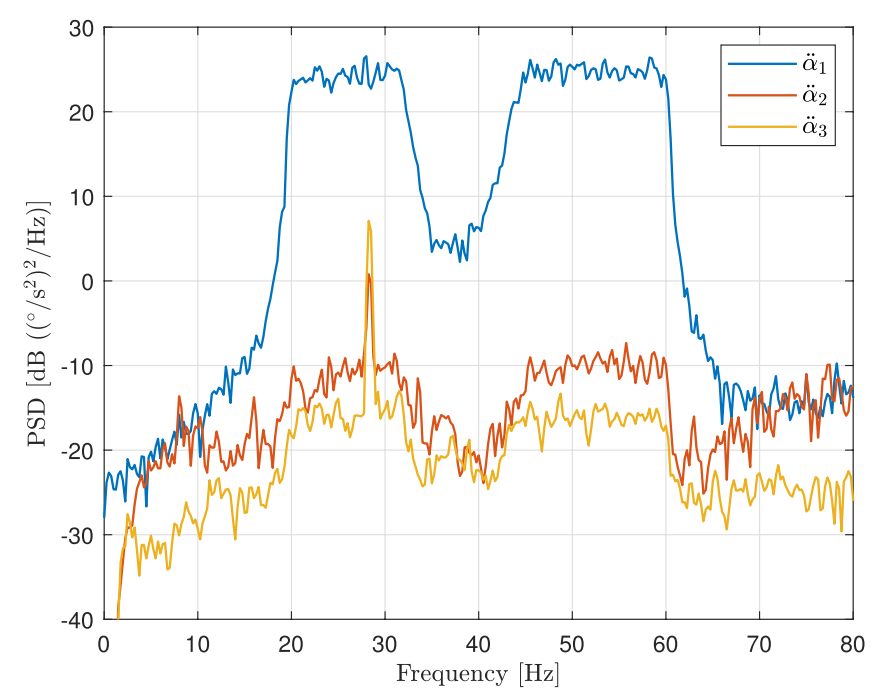

(b)

Fig. 7. PSD in acceleration of the 6 DOFs of the rotor base for the random excitation: (a) pure translational DOFs and (b) angular DOFs.

of the shaft speed of rotation, mostly present experimentally, for instance at $14.2 \mathrm{~Hz}(0.5 \mathrm{X})$ or $85 \mathrm{~Hz}(3 \mathrm{X})$. These deviations may be explained by certain asymmetric properties either in the shaft or disks, nonlinearities in the hydrodynamic bearings, cyclic phenomena in the motor (ball bearings), etc. Lastly, the whirl of all the orbits is mainly forward since the peaks at $+28.3 \mathrm{~Hz}(1 \mathrm{X})$, $+42.5 \mathrm{~Hz}(1.5 \mathrm{X})$ and $+56.7 \mathrm{~Hz}(2 \mathrm{X})$ are higher than those at the corresponding negative frequencies.

\subsection{Multi-axial random excitation}

In this section, the numerical model is tested with more general motions of the random type. Both bending and axial dynamics are assessed here. The shaker's motion is in-plane with only three DOFs involved: the translation along $\vec{X}_{0}$, the translation along $\vec{Y}_{0}$ and the pitch $\alpha_{1}$ around $\vec{Z}_{0}$. The same power spectral density (PSD) is built for the only two mono-axial accelerometers involved in the driving process of the 6-DOF shaker, with a theoretically nil cross-correlation between each other. This PSD is defined by two plateaus at $2.51 \times 10^{-4} \mathrm{~g}^{2} / \mathrm{Hz}$ in $[20-31.6] \mathrm{Hz}$ and $[45-60] \mathrm{Hz}$ and a third one at $2.51 \times 10^{-6} \mathrm{~g}^{2} / \mathrm{Hz}$ in [35-40] Hz. This specific profile permits avoiding the resonance of the axial mode and the first two bending modes (rows 1, 3 and 4 of Tab. 4) so that the rotor response remains small enough and thus linear. The PSDs in acceleration of the corresponding six DOFs of the shaker, obtained by the process described in [34] and computed on the random part of the test between $5 \mathrm{~s}$ and $19 \mathrm{~s}$ (for a full test occurring between $0 \mathrm{~s}$ and $22 \mathrm{~s}$ ), are presented in $\mathrm{dB}$ in Figure $7 \mathrm{a}$ for $\left(\ddot{X}_{0, t r}, \ddot{Y}_{0, t r}, \ddot{Z}_{0, t r}\right)$ and Figure $7 \mathrm{~b}$ for $\left(\ddot{\alpha}_{1}, \ddot{\alpha}_{2}, \ddot{\alpha}_{3}\right)$. The real base motion is mainly composed of the three targeted DOFs. The peak around $28.3 \mathrm{~Hz}$ present in the other three DOFs $\left(\ddot{Z}_{0, t r}, \ddot{\alpha}_{2}, \ddot{\alpha}_{3}\right)$ corresponds to the shaft frequency of rotation which spreads in the rotor base, but remains low enough.
The numerical and experimental time-history responses in $(u, w)$ for Node 15 and in $-v$ (the minus sign is because the axial sensor is directed along $-\vec{y}$ ) for Node 28 are presented in Figure 8 in a short time interval for the sake of clarity. The corresponding PSDs, computed in the random part of the test in $t \in[5 ; 19] \mathrm{s}$, are depicted in Figure 9. Firstly, as expected, the amplitude of $u$ in Figure 8 shows random variations while the amplitude of $w$ is quite constant and unaffected by the base excitation, which is in agreement with the level of the corresponding translational base accelerations $\ddot{X}_{0, t r}$ and $\ddot{Z}_{0, t r}$ in Figure 7a. Secondly, as for the harmonic case of Section 3.2, the amplitudes of $u$ and especially $w$ are slightly overestimated numerically. This overestimation indeed mostly occurs at the shaft frequency of rotation (see Fig. 9) in response to the residual mass unbalance, as in the previous harmonic case. A small peak around $10.4 \mathrm{~Hz}$, which is related to the first torsion mode, is predicted numerically because of a coupling between bending and torsion in the damping matrix $\mathbf{C}_{\mathbf{j b}}$. Nonetheless, since this peak does not appear experimentally, this coupling is likely to be lower than expected. Another peak around $56.7 \mathrm{~Hz}(2 \mathrm{X})$ is found in the measurements but is not predicted as the model considers only an axisymmetric shaft and disks. Regarding the axial displacement, remarkable agreement is seen in the whole frequency range of the study. In this frequency range, the shaft responds on its first axial rigid-body mode in the same way as for a 1-DOF mass-damper-spring system (all the deformation acts on the motor-shaft coupling).

\subsection{Mono-axial chirp sine excitation}

So far, only the bending and axial shaft dynamics have been evaluated. The encoders were not installed in the previous cases since they may alter the original shaft 


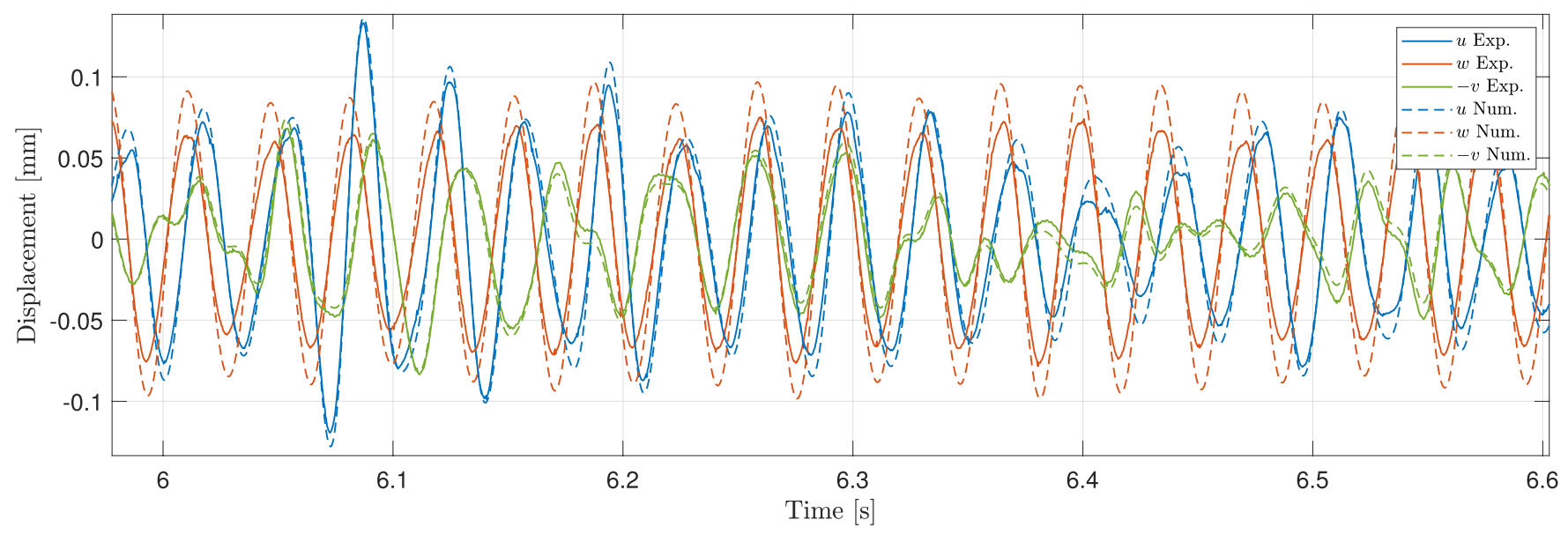

Fig. 8. Experimental (Exp.) and numerical (Num.) comparisons of displacements $(u, w)$ of Node 15 and $-v$ of Node 28 for a random base excitation.

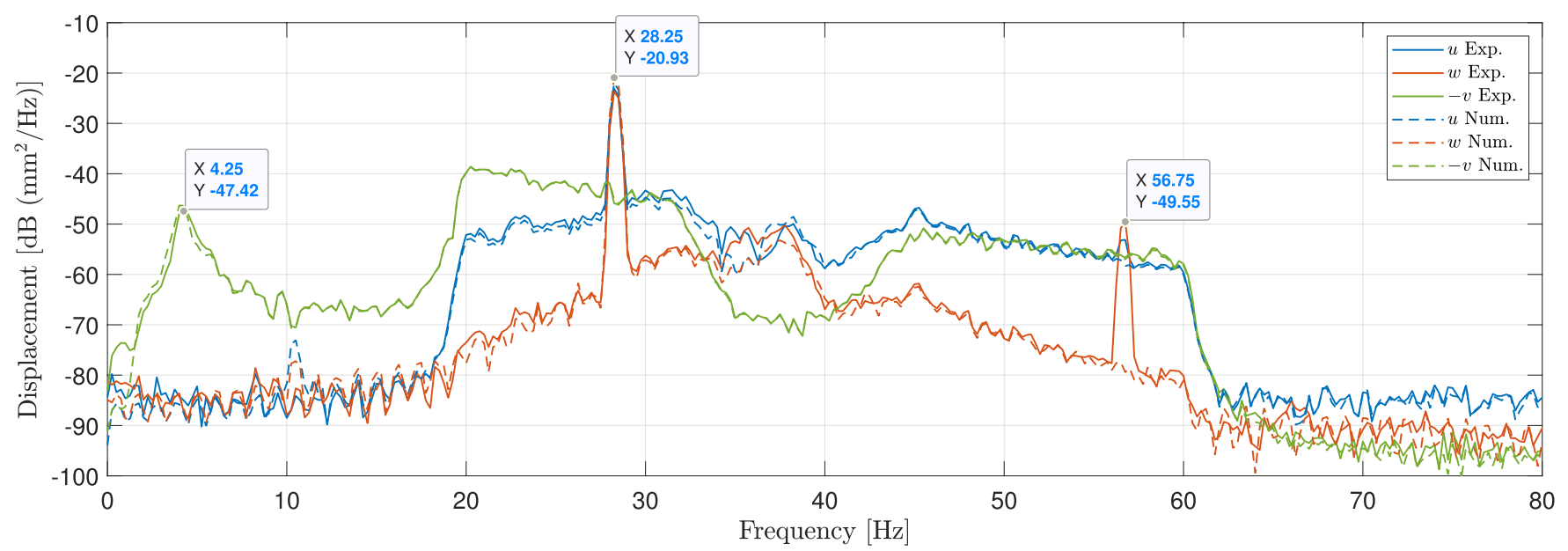

Fig. 9. PSD of the time-history responses of Figure 8 computed for $t \in[5 ; 19] \mathrm{s}$.

dynamics and introduce additional sources of uncertainties. For instance, the balancing is perturbed, the axial rigid-body mode is deeply damped and several harmonics of the shaft speed of rotation appear due in particular to the ball bearings inside the encoders. In this context, a test focusing only on the torsion dynamics was conducted. Consequently, a mono-axial roll (around $\vec{y}$ ) of the rotor base was created, with a targeted instantaneous axis of rotation defined to be coaxial with the shaft axis of rotation (i.e. with $x_{I}=y_{I}=z_{I}=0$ ) in order to minimize the bending and axial responses. However, maintaining this coaxiality efficiently with a random excitation profile through the shaker driving process is quite difficult. Moreover, a mono-frequency harmonic excitation does not permit distinguishing the torsional shaft response from the bending one precisely. For these reasons, a chirp sine profile of the base rotation was chosen.

Since the first torsional mode is almost a pure coupling mode with no shaft deformation, it can hardly be detected by the encoders both mounted on the shaft. Therefore, the second torsional mode was targeted in this study. To this end, the roll rotation is defined by an angle
$\alpha_{3}=A_{3}(t) \cos \left(2 \pi f_{i} \frac{k^{t}-1}{\ln k}\right)$ with $k=\left(f_{f} / f_{i}\right)^{1 / T}, f_{i}=10 \mathrm{~Hz}$ the initial frequency, $f_{f}=125 \mathrm{~Hz}$ the final frequency and $T=16 \mathrm{~s}$. In this way, the instantaneous frequency $f_{3}(t)=f_{i} k^{t}$ of the roll rotation increases exponentially from $f_{i}$ at $t=0$ to $f_{f}$ at $t=T$. In order to maintain a relatively constant amplitude of angular acceleration, $A_{3}$ is defined so that it decreases with time such that $A_{3}(t)=A_{0} \frac{1}{\left(2 \pi f_{3}(t)\right)^{2}}$ with $A_{0}=1200^{\circ} / \mathrm{s}^{2}$. The time variations of the targeted and real (experimental) $\alpha_{3}$ are presented in Figure 10. Despite a real amplitude slightly higher than expected, it can be seen that the chirp sine profile is globally satisfactory. The other real DOFs of the shaker, not shown here, are also present in practice with maximum accelerations around $0.15 \mathrm{~g}, 0.25 \mathrm{~g}, 0.2 \mathrm{~g}$, $250^{\circ} / \mathrm{s}^{2}, 60^{\circ} / \mathrm{s}^{2}$ for $\ddot{X}_{0, t r}, \ddot{Y}_{0, t r}, \ddot{Z}_{0, t r}, \ddot{\alpha}_{1}$ and $\ddot{\alpha}_{2}$, respectively. Although they are not nil, they are not high enough to perturb the mono-axial motion.

To remove the rigid-body rotation $\phi^{*}$ of the shaft and emphasize the torsional dynamics, the experimental response is established in terms of an angle $B$ obtained as the difference of the total shaft angle of rotation $\phi$ between 


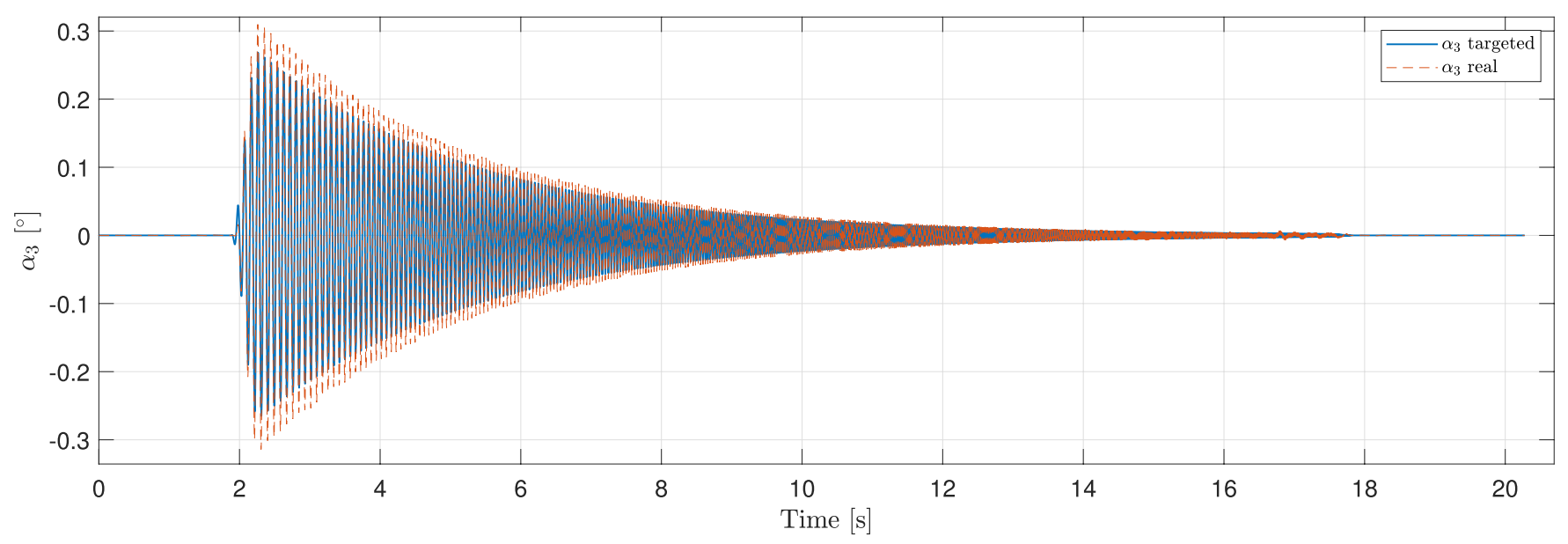

Fig. 10. Comparison between the targeted and real roll angle $\alpha_{3}$ of the rotor base with a chirp sine profile.

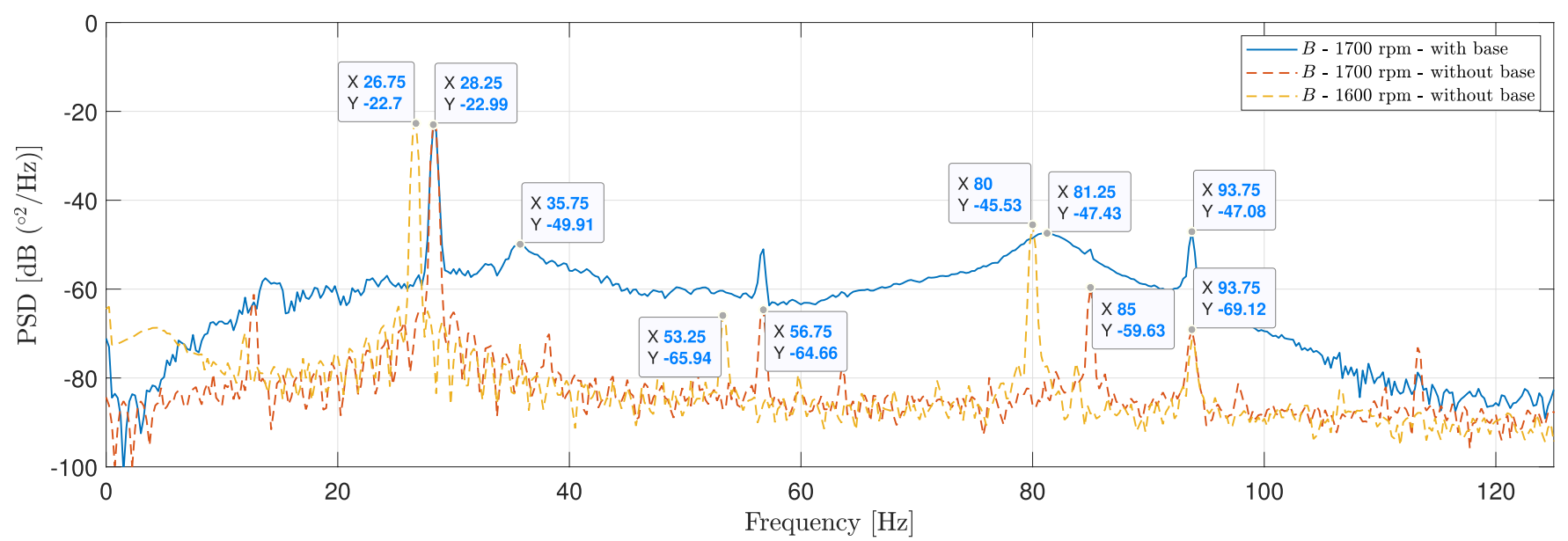

Fig. 11. Experimental comparison of angle $B=\beta_{27}-\beta_{2}$ with and without base roll excitation in chirp sine.

Nodes 27 and 2 (namely $B=\phi_{27}-\phi_{2}=\beta_{27}-\beta_{2}$ ) where the two encoders are located. Moreover, a high-pfilter with a cut-off frequency of $5 \mathrm{~Hz}$ is applied to the experimental angle $B$. The corresponding result is presented in Figure 11 in terms of its PSD, computed between $2.3 \mathrm{~s}$ and $17.4 \mathrm{~s}$. The response of angle $B$ is also plotted without base motion for two different shaft speeds of rotation to emphasize the impact of the base on the torsional response. The two corresponding PSDs are computed from the same time duration of $15 \mathrm{~s}$ as for the PSD with base motion.

Firstly, it is noteworthy that phenomena related to bending dynamics were observed experimentally around $28.25 \mathrm{~Hz}$ and $56.75 \mathrm{~Hz}$, which correspond to the harmonics $1 \mathrm{X}$ and $2 \mathrm{X}$ of the mass unbalance forces, and around $35.75 \mathrm{~Hz}$ and $81.25 \mathrm{~Hz}$, which correspond to Modes 4 and 5 of Table 4 (with the encoders). These frequencies, that should, theoretically, not be detected by the encoders, are present since the shaft deflections inherently induce a rotational motion of the stator part of the encoders (see Sect. 3.1 for more details). Secondly, a peak at $93.75 \mathrm{~Hz}$ related to the second torsional mode appears. This peak is not related to any bending phenomena, because it is missing from the measured radial deflection, as seen for instance in Figure 6, and it is insensitive to the speed of rotation as seen with the dashed lines of Figure 11 in the case of no base excitation. Thus, it can be stated that it is related to the second torsion mode, well predicted by the model, see Mode 7 at $92.50 \mathrm{~Hz}$ in Table 4 . On the other hand, erasing the base excitation leads to an attenuation of $-22 \mathrm{~dB}$ on the $93.75 \mathrm{~Hz}$ torsion peak: the base roll excitation magnifies the torsional behavior of the rotor-bearing system.

\section{Conclusion}

This work addressed the experimental validation of an on-board rotor model supported by finite-length hydrodynamic bearings and subject to a wide variety of excitations, such as translations and/or rotations, monoor multi-axial, harmonic or random or in chirp. To this end, a rotor test bench was designed, built, instrumented and mounted on a 6-DOF hydraulic shaker. The real excitations produced by the latter were introduced in the FEM by solving an inverse problem to obtain better concordance between the calculated and measured results. All the shaft dynamics, i.e. bending, torsion and 
axial motions, were assessed. Excellent agreements were obtained in terms of shaft orbits and full spectrums for the bending dynamics with the multi-axial harmonic base excitation. However, some slight discrepancies in the amplitudes were noted and could mainly be assigned to the presence of residual mass unbalance in the experiment and possibly to certain limitations in the hydrodynamic bearing modeling (temperature and viscosity variations in the fluid, angular misalignment, bearing roughness, etc.). The axial dynamics was predicted with great accuracy in both the time and frequency domains, even in a complex case of random excitation. Regarding torsion, a roll base rotation in chirp sine permitted demonstrating experimentally the response of the second mode, whose frequency matched that of the model.

\section{Conflict of interest}

None.

\section{Acknowledgements}

This work was supported by the French National Research Agency (ANR) in the framework of ANR-Labcom-SME AdViTAM, 16-LCV1-0006 and Equipex PHARE 10-EQPX-0043.

\section{References}

[1] J. Zhao, I. Linnett, L. McLean, Stability and bifurcation of unbalanced response of a squeeze film damped flexible rotor, J. Tribol. 116, 361-368 (1994)

[2] J. Inayat-Hussain, H. Kanki, N. Mureithi, On the bifurcations of a rigid rotor response in squeeze-film dampers, J. Fluids Struct. 17, 433-459 (2003)

[3] H. Ma, F. Yin, Y. Guo, X. Tai, B. Wen, A review on dynamic characteristics of blade-casing rubbing, Nonlinear Dyn. 84, 437-472 (2016)

[4] M. Dakel, S. Baguet, R. Dufour, Steady-state dynamic behavior of an on-board rotor under combined base motions, J. Vib. Control 20, 2254-2287 (2014)

[5] M. Dakel, S. Baguet, R. Dufour, Nonlinear dynamics of a support-excited flexible rotor with hydrodynamic journal bearings, J. Sound Vib. 333, 2774-2799 (2014)

[6] Q. Han, F. Chu, Dynamic behaviors of a geared rotor system under time-periodic base angular motions, Mech. Mach. Theory 78, 1-14 (2014)

[7] Q. Han, F. Chu, Parametric instability of flexible rotorbearing system under time-periodic base angular motions, Appl. Math. Model. 39, 4511-4522 (2015)

[8] R. Bouziani, N. Ouelaa, Simulation of the dynamic behavior of a rotor subject to base motion under variable rotational speed, Mech. Ind. 18, 308 (2017)

[9] A. Saimi, A. Hadjoui, An engineering application of the hp version of the finite elements method to the dynamics analysis of a symmetrical on-board rotor, Eur. J. Comput. Mech. 25, 388-416 (2016)

[10] R. Wang, X. Guo, Y. Wang, Nonlinear analysis of rotor system supported by oil lubricated bearings subjected to base movements, Proc. Inst. Mech. Eng. C 230, 543-558 (2016)
[11] M.R. Reddy, J. Srinivas, Vibration analysis of a support excited rotor system with hydrodynamic journal bearings, Proc. Eng. 144, 825-832 (2016)

[12] Z. Liu, Z. Liu, Y. Li, G. Zhang, Dynamics response of an on-board rotor supported on modified oil-film force considering base motion, Proc. Inst. Mech. Eng. C 232, 245-259 (2018)

[13] F. Vicencio, E.F. Cruz, A high order nonlinear study to evaluate the seismic response of rotating machinesstructure-soil foundation systems, J. Earthquake Eng. 0, 1-33 (2019)

[14] H. Zhu, W. Chen, R. Zhu, J. Gao, M. Liao, Study on the dynamic characteristics of a rotor bearing system with damping rings subjected to base vibration, J. Vib. Eng. Technolog. 8, 121-132 (2020)

[15] T.d.P. Sales, E. Spuldaro, L.F. Damy, D.A. Rade, Dynamic modeling of flexible rotors mounted on an elastic base undergoing arbitrary attitude motion, in: International Conference on Rotor Dynamics. (Springer, 2018), pp. $562-576$

[16] T. Soni, J.K. Dutt, A. Das, Parametric stability analysis of active magnetic bearing-supported rotor system with a novel control law subject to periodic base motion, IEEE Trans. Ind. Electr. 67, 1160-1170 (2019)

[17] T. Soni, A. Das, J. Dutt, Active vibration control of ship mounted flexible rotor-shaft-bearing system during seakeeping, J. Sound Vib. 467, 115046 (2020)

[18] H. Phadatare, B. Choudhary, B. Pratiher, Evaluation of nonlinear responses and bifurcation of a rotor-bearing system mounted on moving platform, Nonlinear Dyn. 90, 493-511 (2017)

[19] M. Shahgholi, G. Payganeh, Forced vibrations of nonlinear symmetrical and asymmetrical rotating shafts mounted on a moving base, ZAMM J. Appl. Math. Mech. 99, e201700097 (2019)

[20] Y. Yi, Z. Qiu, Q. Han, The effect of time-periodic base angular motions upon dynamic response of asymmetric rotor systems, Adv. Mech. Eng. 10, 1687814018767172 (2018)

[21] X. Qiu, Q. Han, F. Chu, Dynamic modeling and analysis of the planetary gear under pitching base motion, Int. J. Mech. Sci. 141, 31-45 (2018)

[22] M. Sousa, V. Del Claro, A. Cavalini, V. Steffen, Numerical investigation on the dynamic behavior of an onboard rotor system by using the fem approach, J. Braz. Soc. Mech. Sci. Eng. 39, 2447-2458 (2017)

[23] C.M. Stanica, M.V. Predoi, I. Stroe, Study of rotating machineries in a non-inertial reference frame subjected to rotations, Romanian J. Acoustics Vib. 16, 125-136 (2019)

[24] Y. Han, M. Li, Nonlinear dynamic characteristics of marine rotor-bearing system under heaving motion, Shock Vib. 2019 (2019)

[25] W. Zhao, M. Li, Y. Liu, Nonlinear dynamics of marine rotor system coupled with air bag-floating raft subjected to the basement excitations in lateral directions, Shock Vib. 2020 (2020)

[26] A.S. Lee, B.O. Kim, Y.-C. Kim, A finite element transient response analysis method of a rotor-bearing system to base shock excitations using the state-space newmark scheme and comparisons with experiments, J. Sound Vib. 297, 595615 (2006)

[27] M. Duchemin, A. Berlioz, G. Ferraris, Dynamic behavior and stability of a rotor under base excitation, J. Vib. Acoustics 128, 576-585 (2006) 
[28] N. Driot, C.H. Lamarque, A. Berlioz, Theoretical and experimental analysis of a base-excited rotor, J. Comput. Nonlinear Dyn. 1, 257-263 (2006)

[29] M. Sousa, V. Del Claro, A. Cavalini, V. Steffen, Experimental validation of an onboard rotor fe model, in: Proceedings of the 24th ABCM International Congress of Mechanical Engineering, COBEM, Curitiba, Parana, Brazil (2017)

[30] L. Chen, J. Wang, Q. Han, F. Chu, Nonlinear dynamic modeling of a simple flexible rotor system subjected to timevariable base motions, J. Sound Vib. 404, 58-83 (2017)

[31] C. Jarroux, J. Mahfoud, R. Dufour, F. Legrand, B. Defoy, T. Alban, Dynamic behavior of a rotor-AMB system due to strong base motions, in International Conference on Rotor Dynamics (Springer, 2018), pp. 340-349

[32] Y. Briend, M. Dakel, E. Chatelet, M.-A. Andrianoely, R. Dufour, S. Baudin, Effect of multi-frequency parametric excitations on the dynamics of on-board rotor-bearing systems, Mech. Mach. Theory 145, 103660 (2020)

[33] T. Zheng, N. Hasebe, Calculation of equilibrium position and dynamic coefficients of a journal bearing using free boundary theory, ASME J. Tribol. 122, 616-621 (2000)

[34] Y. Briend, E. Chatelet, R. Dufour, F. Legrand, S. Baudin, Identification of real translational and rotational displacements of six-axial shakers with only six measured linear accelerations, Mech. Syst. Signal Process. 154, 107584 (2021)

[35] G. Mogenier, T. Baranger, G. Ferraris, R. Dufour, L. Durantay, A criterion for mode shape tracking: application to Campbell diagrams, J. Vibrat. Control 20, 179-190 (2014)

[36] P. Goldman, A. Muszynska, Application of full spectrum to rotating machinery diagnostics, Orbit 20, 17-21 (1999)

Cite this article as: Y. Briend, E. Chatelet, R. Dufour, M.-A. Andrianoely, F. Legrand, S. Baudin, Dynamics of on-board rotors on finite-length journal bearings subject to multi-axial and multi-frequency excitations: numerical and experimental investigations, Mechanics \& Industry 22, 35 (2021) 